GENERALIZED HOPF BIFURCATION AND

h-ASYMPTOTIC STABILITY

\author{
by \\ S. R. Bernfeld 1,2 \\ and \\ L. Salvadort ${ }^{3}$ \\ Technical Report No. 122 \\ November, 1979
}

${ }^{1}$ Regearch partially supported by Itallan Counctl of Research (C.N.R.) and U.S. Axmy Research Grant DAAG 29-77-60062.

${ }^{2}$ Department of Mathematica, University of Texas at Arlington, Arlington, Texas and Dipartimento d1 Matematica, Univera1tà d1 Trento, Trento, Italy. ${ }^{3}$ Dipartimento di Matematica, Universita di Trento, Trento, Italy. 


\section{GENERALIZED HOPF BIFURCATION AND \\ h-ASYMPTOTIC STABILITY}

\section{Introduction}

The prevalent approach to the Hopf bifurcation problem is to prove directly the existence of the bifurcating perlodic orbits by using such atandard procedures as the 1mp11c1t function theorem, the LlapunovSchmidt method and 1 ts known variants, and topological degree arguments (see [7]). The phenomenon of Hopf bifurcation often occurs because of exchange of stability properties of the equilibrium under perturbations (see for Instance, Chafee in [7] p. 85-88, Andronov et. a1. [1], Marchetti et. a1. [6] and Negrint and Sa1vadori [8]). This connection between the exchange of stability of the equilibrium and the appearance of bifurcating periodic orbits can be carefully Investigated in order to develop a different approach for obtaining existence results and qualitative properties of these orbits. Now we want to provide a systematic development of the procedure sketched in [6] and [8] by considering the generalized Hopf bifurcation as was studied by Chafee [3] who used the alternative method as described by Hale [4].

In particular consider an $n$ dimensional system of differential equations

$$
\dot{x}=f_{0}(x)
$$

where $f_{0} \in C^{\infty}\left[B^{n}\left(x_{0}\right), R^{n}\right], f_{0}(0)=0$, and $B^{n}\left(r_{0}\right)=\left\{x \quad R^{n}:\|x\|<x_{0}\right\}$. 
Aasume the Jacoblan matrix $f_{0}{ }^{\prime}(0)$ has a complex confugate palr of elgenvalues \pm 1 and that all other elgenvalues $\lambda$ satisfy $\lambda \neq m 1$, $m=0, \pm 1, \pm 2, \ldots$.

For those $f$ close to $E_{0}$ consider the perturbed differential equation

$$
\dot{x}=f(x) \text {. }
$$

We are interested in determining the number of nonzero perlodic orbits of (1.2) lying near the origin and having perlod $T$ close to $2 \pi$ for each $f$ close to $F_{0}$ (with respect to an appropriate topology). In constderling this problem, Chafee [3] constructed a determining equation $\psi(\xi, f)=0$ where $\xi=\rho^{2}$, In which $\rho$ is a measure of the amplitude of the periodic solution being sought and $f$ is the function appearing in (1.2). Letting $k$ (EInfte) be the mu1tiplicity of the root $\xi=0$ of the equation $\psi\left(*, f_{0}\right)=0\left(\psi\left(0, f_{0}\right)=0\right)$, Chafee proved among other results the following two properties: (a) there exists a nelghborhood $N$ of $f_{0}$ and number $r_{1}>0$ such that for any $E N$ equation (1.2) has no more then $k$ nontrivial periodfc orbits in $B^{n}\left(r_{1}\right)$ with period close to $2 \pi$; (b) for any Integer $1,0 \leq j \leq k$, for any netghborhood $N^{*}$ of $f_{0}, N^{*} \subseteq N$, and for any $r_{2} \in\left(0, r_{1}\right]$ there exists an $f \in N^{*}$ ouch that equation (1.2) has exactly $f$ nontrivial periodic orbits in $\mathrm{B}^{\mathrm{n}}\left(\mathrm{r}_{2}\right)$ with pertodic close to $2 \pi$.

since the construction of the determining equation requires the use of fixed point theorems its form is only known implicitly. Thus the 
determination of the number $k$ is a new problem that needs to be solved. We are interested in solving this new problen.

In this paper we 11lustrate the case $n=2$. The general problem Is much more difficult and a paper devored to the general case in collaboration with $P$. Negrint fs in preparation. The techniques in $\mathrm{R}^{\mathrm{n}}$ are extenglons of the tdea of this paper in $\mathrm{R}^{2}$. Indeed the use of approplate Llapunov functons used in this paper wh some dellcate modiflcations allows to treatment in $\mathrm{K}^{\mathrm{k}}$. We prove that properties (a) and (b) occur if and only it the origin of (1.1) is efther $(2 k+1)$ asymptotical1y table or $(2 k+1)$ completely unstable; that is the origin is asymptotically gtable in the future or in the past and this property 19 recognizable fin a sutable sense by the terms of $f_{0}(\cdot)$ of degree $\leq 2 k+1$. This result glves a complete answer to the problem since we provide a constructive procedure to determine $k$. Indeed this to exactiy the classtcal method of Polncare which is an algebraic procedure to construct efther a power geries atiafylng formaliy the condition of betng a fixst lntegral of (1.1) or a postlve defintte function whase dertvatives along the solucton of (1.1) Is defintte in sign. Now let $S_{1}, 0 \leq 1 \leq k$, be those $E \in N$ for which system $(2.2)$ has exactly $I$ nontrivial perfodic orbits lylng in $B^{2}\left(r_{1}\right)$. Our techniques allow us to conetruct a broad class of functons lying in the Interior of $s_{f}$. Indeed these functions can be found by determining polynomials of order $k$ that have $f$ almple roots. In this way we provide a method for determintrg a clase of functions that enfoy the structura1 property of preserving the number of perlodtc orblta near the origin under mall perturbation (see the Remarks after the necessity 
part of the proof of theoren 3.1 ).

Uaing different techiques Andronow and Leontowich in Chapter IX of [1] previousiy obtalned the propartids (a) and (b) occur if the ortg1n of (1.1) is efther $(2 k+1)$-agymptotcally stable or $(2 k+1)-$ completely unstable. Their techniques do not seem to provide a constructive procedure cot she determination of those sthen have the above structurat property.

For the care $k=1$ wa have provided an answer to a problem posed by Chafee [3, sec, 7]. Namely, we have glven all explic1t condition, based on the Polncare procsdure to deterthe whether there extsts one pertadic or no pertodic orblta of $(1.2)$.

We aloo constder the case $k$ and obtain an sufinte dimensional analogue of our previous reatita.

The methods we employ here involve the ratatonghp between the sign of the firat non-nero derivatue of the displacentent function of (1.1) at the origin and the above stability properties of the origin. In contrast to Andronov and Leontovich we ind it uaful to put (1.2) into a type of notmal form (see Takens (10) so that the polynonlal part of the displacem ment function 18 in a $1-1$ correspondence with the first $2 k+1$ terms of the Maclaurin expanston of the rghthand olde of the normal Eorm.

In fact out paper ghoss that the generajtad hopf btfurcation in $R^{2}$ reduces to a scudy of a Entto dinengional varglng vector Eleld. Thus If

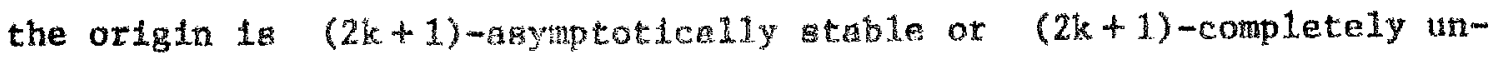
atable far syster (1.1) then in the language of Takens [10] our problem reduces to a gtudy of 2 to 1 untveragl unfoldinge. 
Some of our results without proofs were given at a conference in Triegte [2].

Finaliy we remark that this paper 111ustrates the Importance of the concepts and arguments of stab111ty theory in approaching hopf bifurcat1on. 


\section{Notatlon and Preliminaries}

Under the hypotheses given on $f_{0}$, by applying a coordinate transformation, equation (1.1) in $R^{2}$ can be written as

$$
\begin{aligned}
& \dot{x}=-y+x_{0}(x, y), \\
& \dot{y}=x+y_{0}(x, y),
\end{aligned}
$$

where $X_{0}(0,0)=Y_{0}(0,0)=0$ and $X_{0}^{\prime}(0,0)=Y_{0}^{\prime}(0,0)=0$. We sha11 again refer to the right-hand side of $(2.1)$ as $f_{0}$. Simllarly equation (1.2) can be written as

$$
\begin{aligned}
& \dot{x}=\alpha x-B y+X(x, y), \\
& \dot{y}=\alpha y-\beta x+Y(x, y),
\end{aligned}
$$

where $X(0,0)=Y(0,0)=0$ and $X^{\prime}(0,0)=Y^{\prime}(0,0)=0$ and again refer to the right-hand side of $(2.2)$ as $f$.

We now define the topology on the space $C^{\infty}\left[B^{2}\left(r_{0}\right), R^{2}\right]$, where $r_{0}$ Is a fixed real positive number and $B^{2}\left(r_{0}\right)$ is the open ball in $R^{2}$ centered at the origin having radius $x_{0}$. As in [3] define function \|\| mapping $C^{\infty}\left[B^{2}\left(r_{0}\right), R^{2}\right]$ tnto $R$ by

$$
\|E\|=\sum_{x=0}^{\infty} \frac{1}{2^{l}} \frac{\|f\|^{(l)}}{1+\|f\|^{(l)}}
$$

where $\|f\|^{(R)}$ denotes the usut $1 c^{l}$-gupremum norm of $f$ on $B^{2}\left(r_{0}\right)$. 
Then $C^{\infty}\left[B^{2}\left(x_{0}\right), R^{2}\right\rfloor$ is a metric 11near space under \|\| . Converting (2.2) Into polax form by letting $x=r \cos \theta, y=r \sin \theta$, we have

$$
\begin{aligned}
& \dot{r}=a r+x^{*}(r, \theta) \cos \theta+y^{*}(r, \theta) \sin \theta, \\
& r \dot{\theta}=\beta r+y^{*}(x, \theta) \cos \theta-x^{*}(r, \theta) \sin \theta,
\end{aligned}
$$

where $X^{*}(x, \theta)=X(r \cos \theta, r \sin \theta)$ and $Y^{*}(r, \theta)=X(x \cos \theta, r \sin \theta)$. since $\beta$ has value close to one and $X^{*}(x, \theta), Y^{*}(x, \theta)$ are $O(x)$ we have the exigtence of $\bar{r}>0$ and $b>0$ auch that $\dot{\theta}>b$ for a11 $r \in[0, \bar{r}]$. For every $r^{0} \in(0, \bar{r})$ and $\theta_{0} \in R$ the orbtt of (2.2) pasalng through $\left(\mathrm{r}^{0}, \theta_{0}\right)$ w111 be represented by the solution $r\left(E, \theta, r^{0}, \theta_{0}\right)$ of

$$
\frac{d r}{d \theta}=R(e, r, \theta), r\left(\theta_{0}\right)=r^{0}
$$

where $R(f, r, \theta)=\frac{a r+x^{*}(r, \theta) \cos \theta+x^{*}(r, \theta) \sin \theta}{\theta(r, r, \theta)}$

wth $\theta(f, r, \theta)-\frac{x^{*}(r, \theta) \cos \theta-x^{*}(r, \theta) \operatorname{tn} \theta}{r}$

for $x>0, \theta(f, 0, \theta) . \beta$. By continuous dependence arguments it follows that for a sufficiently anall netghborhood $M$ of $f_{0}$ and for suffictently amal1 $\bar{c}>0$ the solution $r(r, \theta, c, 0)$ of $(2.3)$ exists on $[0,2 \pi]$ for ald $f \in M$ and $A 11$ c $\in[0, \bar{c})$.

We now define the displacement function for $(2.2), v(f, c)$, given by 


$$
V(E, c)=r(E, 2 \pi, c)-c
$$

where $r(f, 2 \pi, c) \equiv r(f, 2 \pi, c, 0)$. Stuce $R(f, *, \cdot) \in C^{\infty}$ we have

$$
r(f, \theta, c)=u_{1}(f, \theta) c+u_{2}(x, \theta) c^{2}+\ldots u_{k}(r, \theta) c^{k}+n(f, \theta, c)
$$

where $k$ is any postlve integer and $n(f, \theta, *)$ is of order greater than $k$; moreover, $u_{1}(f, 0)=1, u_{\ell}(f, 0)=0,1=2,3, \ldots, k$, and $\eta(f, 0, c)=0$. Insert1ng (2.4) tnto $(2.3)$, we have for each flxed $f$ the following systen of differential equations for $u_{1}(f, \theta)$;

$$
\begin{aligned}
& \frac{\partial u_{1}}{\partial \theta}=\frac{\alpha}{\beta} u_{1} \\
& \frac{\partial u_{1}}{\partial \theta}=\frac{\alpha}{\beta} u_{1}+v_{1}\left(u_{1}, u_{2}, \ldots, u_{1-1}, \theta\right), 1=2,3, \ldots .
\end{aligned}
$$

Defining $\tilde{V}(f, c) \equiv \frac{V(f, c)}{c}$ for $c \neq 0$ and $\tilde{V}(f, 0)=u_{1}(f, 2 \pi)-1$, we see that the orbit of $(2.3)$ through $(c, 0)$ la closed if and only if $V(F, c)=0$; that is either $c=0$ or $\tilde{V}(f, c)=0$. Notice that $V(f, \cdot)$ and $\tilde{V}(f, \cdot)$ are $C^{\infty}$.

We not fntroduce the concept of h-asymptotlc atability which plays a major role in the formulation of our regults. The following terminology and results were given in the paper of Negrind and Salvadorl [8].

Definition. Let $h$ be an Integer $\geq 2$. The solution $x \equiv y \equiv 0$ of (2.1) is sald to be h-asymptotudelly atable (reap. h-completely unstable) 
if

(1) For every $\tau_{1}, \tau_{2} \in C^{o n}\left[B^{2}\left(t_{0}\right), R\right]$ of order greater than $h$ the golution of the gystem

$$
\begin{aligned}
& \dot{x}=-y+x_{02}(x, y)+x_{03}(x, y)+\ldots x_{0 h}(x, y)+\tau_{1}(x, y) \\
& \dot{y}=x+y_{02}(x, y)+y_{03}(x, y)+\ldots y_{0 h}(x, y)+\tau_{2}(x, y)
\end{aligned}
$$

ta asymptotlcally table (resp. completely unstable). Here $x_{01}, Y_{01}, 2 \leq 1 \leq h$, represent the $1^{\text {th }}$ terms of the Maclaurin expanation of $X_{0}, Y_{0}$ respectively (recall that $\left.x_{01}(x, y) \equiv y_{01}(x, y) \equiv 0\right)$;

(i1) property (1) ts not antisfled when $h$ is replaced by any Integer $n \in\{2,3, \ldots, h-1\}$.

The properties in the above defintton can occur only when $h$ ia odd. Moreover it has been ghow that the h-agymptote atability or h-complete instablitty can be recognized by means of a clasafcal procedure due to Polncare [9]. In partlcular conslder a polynomal of the form

$$
F(x, y)=x^{2}+y^{2}+F_{3}(x, y)+\ldots F_{n}(x, y)
$$

where $m$ is an even lnteger and $F_{f}$ is a homogeneous polynomial of degree 1 . Let $\dot{F}_{(2,1)^{(x, y)}}$ be the derivative of $F$ along solutions of (2.1). The Polncare procedure 1 an algebralc nethod for the determination of $m, F$, and a constant $g_{m}$ such that 


$$
\dot{\bar{F}}(2,1)(x, y)=G_{n}^{\left(x^{2}+y^{2}\right)^{m / 2}+x(x, y)}
$$

where $x$ is of ordet greater than o clearly (2.7) is atiafied for m=2 since $G_{2}=0$. In addition 1 (2.7) to satigfied for m with with

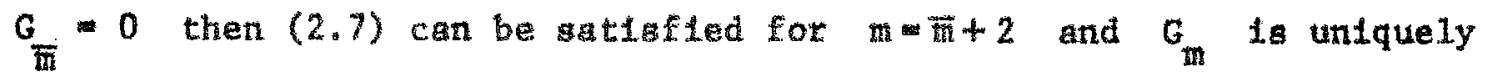
determined.

We now have the followlng relationghip between the Polncare procedure and the h-asymptotic stablilty and h-complete otability of the orfgln of aystem $(2.1)$;

Phoposition 2.1 [8]. Let $h \geq 3$ be an add integer. The solution $x \equiv y \equiv 0$ of (2.1) is h-aymptotically able (resp. h-completely unstable) If and only if $G_{x}=0$ for $x \in\{2,4, \ldots, h-1\}$ and $G_{h+1}<0$ (resp. $\left.G_{h+1}>0\right)$.

Arother result in [8] astabluhes a relationshlp between h-asymptotic stability or h-complete Intablitty of the origin of $(2.1)$ and the diaplacement function of $(2.1)$ evaluated at the or $\lg \ln , V\left(f_{0}, 0\right)$. Namely

Phoposition 2.2[8]. Let $h \geq 3$ be an odd integer. Then the solution $x \equiv y \equiv 0$ of $(2.1)$ s.s has hyptotically scable (resp. h-completely unstable) tf and only if $\frac{\partial^{2} y}{\partial c^{1}}\left(f_{0}, 0\right)$ for $i \in\{1,2, \ldots, h-1\}$ and $\frac{\partial^{h} y}{\partial c^{h}}\left(f_{0}, 0\right)<0 \quad\left(\right.$ resp. $\left.\quad \frac{\partial^{h} y}{\partial c^{h}}\left(f_{0}, 0\right)>0\right)$

Thus the displacement function for (2.1) when the ortgin is h-agymptotically gtable or hacompletely ungtable has the form 


$$
v\left(f_{0}, c\right)=g c^{b}+n\left(E_{0}, c\right)
$$

where $n\left(f_{0}, c\right)$ ts of degree greater tham $h$ in $c$ and the sign of $g$ is the same as the sign of the Polncare constant $G_{h+1}$.

\section{Matn Results}

In this section we preaent our matn results and give the proofs in the next aection. Our fitrst result ig an equivalence between the $h$. asymptotic stablilty or h-complete instability of the origin of (2.1) and the number of non-trivial pertode solutions of (2.2) for those $f$ which are sufficlently close to $f_{0}$.

Theorem 3.1. Let $k$ be any integex such that $k \geq 1$. Then a necessary and auficient condition that the solution $x \equiv y=0$ of $(2.1)$ is elther h-asymptotically stable or h-completely unstable with $h=2 k+1$ 18

(1) there exists a nelghborhood $N$ of $E_{0}$ and a number $r_{1}>0$ such that for any $E \in N$ equation $(2.2)$ has no more than $k$ nontrivial periodic orbtts in $B^{2}\left(r_{1}\right)$;

(1i) for any integer $f, 0 \leq f \leq k$, for any neighborhood $N^{*}$ of E, $N^{*} \subseteq N$, and for any $x \in\left(0, z_{1}\right]$ there exlsts an $f \in N^{*}$ such that equation (2.2) has exactly I nontrivial pertodic orbits in $3^{2}\left(r_{2}\right)$.

In adition if the aolution $x \equiv y=0$ of (2.1) is etther h-asymptotically stable or h-completely unstable with $h=2 k+1$ then 
(1if) letting $s_{j} \equiv s_{j}\left(N, r_{1}\right), 0 \leq f \leq k$, be the set of functions $f \in N$ for which there extst exactly $f$ nontrivial pertodtc orbits of (2.2) in $B^{2}\left(r_{1}\right)$, we have that $s_{j}$ has a nonempty Intertor with $f_{0}$ lying on 1 ts boundary and $N=\bigcup_{j=0}^{k} s_{j}$;

(Iv) for any $t \in\left(0, r_{1}\right]$, there exiata a netghborhood $N_{r} \subseteq N$ of $f$ such that if $f \in N_{r}$ and if $\Gamma$ if a pertodic orbit of (2.2) Iytug in $\mathrm{B}^{2}\left(\mathrm{r}_{1}\right)$ then $\Gamma$ lies in $\mathrm{B}^{2}(r)$.

Remarks. Chafee [3] obtatned the conclustons $(1)-(1 v)$ under the hypotheses that the determining equation $\psi(\xi, f)=0$ (see the Introduction) satisfles the condition that $\xi=0$ is a zero of multiplicity $k$ of $\psi(\cdot, f)$. Moreover he proved the conclusions held in $\mathrm{R}^{\mathfrak{n}}$ (see the Introduction) for those orbits whose perfod is near $2 \pi$. In $\mathbb{R}^{2}$ we have by continuous dependence that all the perlodic orbits near the orfgin have pertodtc near $2 \pi$. Moreover it is suffictent that $f_{0}, f \in C^{k}\left[B^{2}\left(x_{0}\right), R^{2}\right]$.

As we mentioned in the Introduction the determination of the multiplictity $k$ of the zeros of $\psi\left(\cdot, f_{0}\right)$ for $\xi=0$ is a new problem in itself ance the construction of $\psi(\xi, f)$ 18 glven frmpleitly. In order to verify the conclustons (1)-(iv) we have provided an expliclt method, which requires the determination of $h$ for which the origin of $(2.1)$ 1.s efther h-asymptotically stable or k-completely unstable. From proposition 2.1 the odd integer $h$ (and thus $k$ since $k=\frac{h-1}{2}$ ) can be found by ut1lizing the classtcal algebrate procedure of polncare.

Due to the equivalence of h-asymptotic stablitty or h-complete Instabluty of the origh for system (2.1) and the conclusion (f) and (11), we gee that our result provides a perclac relationship between the 
extstence of smat1 pertodic orbits of the perturbed equation (2.2) and the stability properties of the origin of system $(2,1)$.

Now we present a reault which extends Theorem 3.1 to the case when $k=\infty$.

Theorem 3.2. A necessary and suffictent condition that the solution $x \equiv y \equiv 0$ of (2.1) Is nether h-agymptotically table nor h-completely unstable for any integer $h$ lo that for any nelghborhood $N$ of $f_{0}$, for any integer $1 \geq 0$, and for any number $x\left[\left(0, x_{1}\right]\right.$ there exists $f \in N$ such that equation $(2.2)$ has I nontrivial periodic solutions lying in $B^{2}(r)$.

For the case $h=3$ our next result gives criterlon, in terms of the Polncare constant $G_{4}$, for establishing whethet $f$ to contalned in $s_{0}$ or $\operatorname{tn} s_{1}$.

Theonem 3.3. Assume that the solution $x \equiv y \equiv 0$ of (2.1.) 1s efther 3-asymptotically stable or 3-completely unstable. Then there exists a nelghborhood $N$ of $f_{0}, N \subseteq N$ and an $\varepsilon \in\left(0, \varepsilon_{1}\right)$ such that we have $E \in S_{0}(N, E)$ if $\alpha G_{4} \geq 0$ and $f \in s_{1}(N, E)$ if $\alpha G_{4}<0$.

Remarks. A andlar result was given by Chatee [3] who proved that $E \in s_{0}(\mathbb{N}, E)$ if $a \psi^{\prime}\left(0, f_{0}\right) \geq 0$ and $E \in s_{1}(N, E)$ if $\alpha \psi^{\prime}\left(0, E_{0}\right)<0$. Chafee potnted out in section 7 of [3] that he has provtded no method for determintug the algn of $\psi^{\prime}\left(0, f_{0}\right)$. From Theoren 3.3 we observe that the algn of $\psi^{*}\left(0, f_{0}\right)$ is the ame as that of $G_{4}$ and we thus have provided an explicit method in texma of $f_{0}$ for the deternination of the conclusions of Theorem 3.3 . 
In the case of the Hapt bffutcation, when the perturbed system 11 restricted to o one parameter family of functions $f_{\mu},-1<\mu<1$ then we find Theorem 3.3 yields results that complement and In a sense Improve some of the work done by Negrind and Salvator1 [8]. In this case $(2.2)$ can be written as

$$
\begin{aligned}
& \dot{x} \alpha(\mu) x-\beta(\mu) y+x(\mu, x, y), \\
& \dot{y} \alpha(\mu) y+B(\mu) x+Y(\mu, x, y),
\end{aligned}
$$

where $\alpha(0)=0, X(0, x, y)=X_{0}(x, y)$ and $Y(0, x, y)=y_{0}(x, y)$. From the $c^{\infty}$ version of the Hopf blfurcation theoren we have that if the "transverality condition" $a^{\prime}(0) \neq 0$ is satiafied then there existo a bifurcation function $\mu(c)$ defined for $c$ nonegative and oma11 auch that for each $c$ and $\mu$ the orb1t of $(3.1)$ through $(c, 0)$ is closed if and only if $\mu$ w(c). If in addition to the transversality condition one assumes that the or 1 in of $(3.1)$ for $\mu=0$ is elther h-asymptotically table or h-completely unstable then Negrint and Salvadorl [8] have proved that the fnverse function $c(u)$ exiats. If, for example, $\alpha^{\prime}(0)>0$ and the origin is h-asymptotlcally atable they prove that for each $\mu$ auficiently andil thete exista a periadic orbit of $(3,1)$. In Theorem 3.3 we prove for $h=3$ the same result whout requiring the condition $\alpha^{\prime}(0)>0$. That is if only ascume $\alpha(\mu)>0$ for $\mu>0$ and that the origtn of $(3.1)$ for $\mu=0$ is 3 -asymptotically stabje then $\alpha(\mu) G_{4}<0$, and thus for each $\mu>0$ there exists perlodic orbit of (3.1) according to Theoren 3.3. Hence the function c(u) exists although $\mu(c)$ nay not exist. 


\section{Proofs}

We lindlcate a procedure that transtorms (2.1) into a normal form developed by Taken $[10]$. Constder a posttive odd integer h $2 k+1$ and let $w\left(w_{1}, \ldots, w_{d}\right)$ be any real d-vector, $d=h^{2}+3 h$. Regard the $w_{1}^{\prime} \mathrm{s}$ as the coeftclents of a two dimenstonat polynomial $Y\left(w, *{ }^{*}\right)$ of degree $h$ and eatiafyting $\Psi(w, 0,0) \equiv 0$. Now consider the function $Z(w, \cdot, \cdot): B^{2}\left(x_{0}\right)+R^{2}$ deflred by

$$
Z(w, x, y)=E_{0}(x, y)+Y(w, z, y)
$$

and the assoclated differental system

$$
\begin{aligned}
& \dot{x}=z_{1}(w, x, y) \\
& \dot{y}=z_{2}(w, x, y)
\end{aligned}
$$

We not1ce that $z(0,0,0)$ and the elgenvalues of the Jacoblan matrix $\mathrm{D}_{\mathrm{x}, \mathrm{y}} \mathrm{z}(0,0,0)$ are \pm 1 . Then, according to the result in $[101$, there exlsts a $C^{\infty}$ diffeomorphlam $T: R^{d} \times R^{2}+R^{d} \times R^{2}$ in whlch $T_{1}(w, x, y)=w_{1}$, $1 \leq 1 \leq d, T_{d+1}(w, x, y)=x_{1}, T_{d+2}(w, x, y)=y_{1}$, such that system $(*)$ can be transformed Into a gystem of the form

$$
\begin{aligned}
& \dot{x}_{1}=-y_{1}+x_{1} g_{1}\left(w_{0} x_{1}^{2}+y_{1}^{2}\right)+x_{1} g_{2}\left(w_{1} x_{1}, y_{1}\right) \\
& \dot{y}_{1}=x_{1}+y_{1} g_{1}\left(w_{1} x_{1}^{2}+y_{1}^{2}\right)+y_{1} g_{2}\left(w_{1} x_{1} y_{2}\right)
\end{aligned}
$$


for $w$ and $(x, y)$ sufficlently stra11, where $g_{1}, g_{2} \in C^{\infty}\left[B^{2}\left(x_{0}\right), R\right]$, $g_{1}(0,0)=0$ and $g_{2} 1 s$ flat at all points $(w, 0,0)$.

We wish to Inplement this transformation on the set of all $f, f(0,0)=0$, in a neighborhood of $E_{0}$. For a given $f$ let $w\left(w_{1}, \ldots, w_{d}\right)$ be the set of coeffictents of the terms of degree $\leq h$ fin the Maclaurdn expangion of $f-f_{0}$. Thus we write

$$
f(x, y)=f_{0}(x, y)+Y(w, x, y)+\tilde{Y}(f, x, y),
$$

where $Y(w, \cdot, \cdot)$ ts a polynomial of degree $h$ satisfying $Y(w, 0,0) \equiv 0$ and $\tilde{Y}$ ts of order $>h$ in $(x, y)$. There by using the above transformation $T$, we easily recognize that $(2.2)$ can be written (we replace $\left(x_{1}, y_{1}\right)$ with $\left.(x, y)\right)$ :

$$
\begin{aligned}
& \dot{x}=a_{0} x-y+a_{1} x\left(x^{2}+y^{2}\right)+a_{2} x\left(x^{2}+y^{2}\right)^{2}+\ldots a_{k} x\left(x^{2}+y^{2}\right)^{k}+q(f, x, y), \\
& \dot{y}=a_{0} y+x+a_{1} y\left(x^{2}+y^{2}\right)+a_{2} y\left(x^{2}+y^{2}\right)^{2}+\ldots a_{k} y\left(x^{2}+y^{2}\right)^{k}+\Psi(f, x, y),
\end{aligned}
$$

where $a_{0}, \ldots, s_{k}$ are constant depending on $f$ (in fact depending only on w). Moteover sgn $a_{0}$ sgn $\alpha$ and $Q, \Psi$ are of order $>2 k+1$ in $(x, y)$.

Suppose the origin for (2.1) ts exther h-asymptotically stable or h-completely ungtable; then

$$
a_{j}\left(f_{0}\right)=0, j=0, \ldots, k-1, a_{k}\left(f_{0}\right)=a,
$$

with a $<0,[$ resp. a $>0$ ] in the case of h-asymptotic stablifty [resp. 
h-complete lostabl11ty). The relations $(4,2)$ are a consequence of the equivalence proved in [8] between the $(8,-)$ [reap. $(8,+)]$ Takens' stngularity and the asaumed h-asymptotic tability [resp. h-complete Instabilityl of the orfgin for $f=f_{0}$. Thus from (4.1) and (4.2) the system (2.1) under the Takens transornation becones

$$
\begin{aligned}
& \dot{x}-y+a x\left(x^{2}+y^{2}\right)^{\frac{h-1}{2}}+\Phi\left(f_{0}, x, y\right) \\
& \dot{y}=x+a y\left(x^{2}+y^{2}\right)^{\frac{h-y}{2}}+\Psi\left(f_{0}, x, y\right)
\end{aligned}
$$

For each $f$ In nelghborhood of $f_{0}$, where $f$ defines the righthand slde of $(2.2)$, 1et $f^{N}$ denote the tight-hand side of $(4.1)$. Since T Is a $C^{\infty}$ diffeomorphism the mapplng $\mathrm{T}_{*}: \mathrm{C}^{\infty}\left[\mathrm{B}^{2}\left(0, \mathrm{r}_{0}\right), \mathrm{R}^{2}\right]$ $\rightarrow C^{\infty}\left[T\left(B^{2}\left(0, r_{0}\right)\right), R^{2}\right]$ defined by $T_{*}(E)$ ta easiy seen to be continuous in the topology given at the begluning of section 2 . Hence $\Phi(\cdot, \cdot, \cdot)$ and $\Psi(\cdot, *, *)$ are continuous.

Thus in order to prove Theorens 3.1 and 3.3 it 19 auffictent to assume that systems $(2.1)$ and $(2.3)$ are In the nomal form given by (4.3) and (4.1) respective1y. In the proof of Theorem 3.1 (11) we need to elaborate further gince the mapping $\mathbb{T}_{*}$ is not a homeomorphiam.

Unless otherwiae stated we sha1l ascume that the orlgin for aystem (2.1) Is efther h-gymptotically stable or h-completely unatable where $h=2 k+1$.

The displacenent function for $(4.1)$, again denoted by $V(f, c)$ Ia glven by 


$$
\begin{aligned}
V(E, c) & \left(u_{1}(E, 2 \pi)-1\right) c+u_{2}(f, 2 \pi) c^{2}+u_{3}(E, 2 \pi) c^{3}+\ldots u_{2 k+1}(E, 2 \pi) c^{2 k+1} \\
+ & \rho(E, c)
\end{aligned}
$$

where $\rho(f *)$ has degree $>2 k+1$. In case $i f_{0}$ then recall $u_{1}\left(\varepsilon_{0}, 2 \pi\right)=1$ and $u_{2 l+1}\left(f_{0}, 2 \pi\right)=0,1=2, \ldots, k-1$. We tha11 again denota by $\tilde{V}(\varepsilon, c)$ the function defined in section 2 .

Proposition 4.1. Consider the oystem (4.1). Then the displacenent function 19 given by

$$
\begin{aligned}
V(f, c) & =\left(u_{1}\left(a_{0}, 2 \pi\right)-1\right) c+u_{3}\left(a_{0}, a_{1}, 2 \pi\right) c^{3} \\
& +\ldots u_{21}+1\left(a_{0}, a_{1}, \ldots, a_{1}, 2 \pi\right) c^{21+1} \\
& +\ldots u_{2 k+1}\left(a_{0}, a_{1}, \ldots, a_{k}, 2 \pi\right) c^{2 k+1}+\rho(E, c)
\end{aligned}
$$

and the relationghtp between $\left(a_{0}, a_{1}, \ldots, a_{k}\right)$ and $\left(u_{1}\left(a_{0}, 2 \pi\right)\right.$, $u_{3}\left(a_{0}, a_{1}, 2 \pi\right), \ldots, u_{21}+1\left(a_{0}, a_{1}, \ldots, a_{1}, 2 \pi\right), \ldots, u_{2 k}+1\left(a_{0}, a_{1}, \ldots, a_{k}, 2 \pi\right)$ is a homeomorphigin (actually a $e^{\infty}$ diffeomorphism) in a neighborhood of $(0,0, \ldots, a)$ such that $u_{1}(0,2 \pi)=1, u_{21}+1(0,0, \ldots, 2 \pi)=0$, $1<1<2 k-1$ and $u_{2 k+1}(0,0, \ldots, 0,2 \pi)=2 \pi a$

Phook: Writing equation $(4.1)$ in polat form we find that

$$
\frac{d x}{d \theta} a_{0} x^{x} a^{3}+\ldots a_{1} x^{21+1}+\ldots a_{k} x^{2 k+1}+\gamma(\theta, x)
$$

where $\gamma(\theta, 2)$ has order greater than $2 k+1$ fin $r$. By asuming $x$ has 
the form $(2.4)$ then $u_{1}(\cdot, \theta)$ satiafles $(2.5)$, which in the care when 1 Is odd reduces to

$$
\begin{aligned}
& \frac{\partial u_{1}}{\partial \theta}=a_{0} u_{1} \\
& \frac{\partial u_{21+1}}{\partial \theta}=a_{0} u_{21+1}+w_{1}\left(a_{1}, a_{2}, \ldots, a_{1-1}, u_{1}, u_{3}, \ldots, u_{21+1}\right) \\
& +a_{1} u_{1}^{21+1} \quad 1=1,2, \ldots, k,
\end{aligned}
$$

where $w_{1} 18$ Inear in each of the $a_{j}, 1,1, \ldots, 1-1$. In the case when 1 is even we elnd that the solution of (2.5) datisfying the Initial conditions $u_{2 j}(f, 0)=0$ le given by $u_{2 j}(f, \theta) \equiv 0$ $f=1, \ldots, k$. We thus see $V(E, c) 18$ glven by $(4,4)$.

By a standard continuous dependence argument it follows that $\left(u_{1}(\cdot, 2 \pi), u_{3}(\cdot, 2 \pi), \ldots, u_{2 k+1}(*, 2 \pi)\right)$ is $c^{\infty}$ in $\left(a_{0}, a_{1}, \ldots, a_{k}\right)$ since the solutions of $(4.5)$ are unfquely determined by initial condtions. since $u_{1}(\cdot, 0)=1, u_{21+1}(\cdot, 0)=0,1, \ldots, k$ and $\varepsilon_{1}\left(0, \ldots, 0, u_{1}, u_{3}\right.$, $\left.\ldots, v_{21-1}\right)=0$ then fxom $(4,5) \quad u_{1}(0, \theta)=1, u_{21+1}(0,0, \ldots, 0, \theta) \equiv 0$ and $u_{2 k+1}(0,0, \ldots, 0, a, \theta)=a \theta$. To complete the proof, the solution

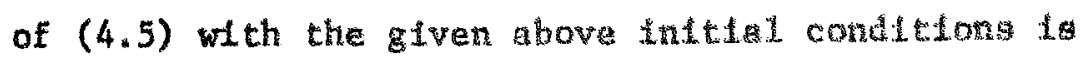

$$
\begin{gathered}
u_{1}(2 \pi)=e^{2 \pi a} \\
u_{21+1}(2 \pi)=e^{2 \pi a} \int_{0}^{2 \pi} e^{-2 \theta} s_{1}\left(a_{1}, a_{2}, \ldots, a_{1-1}, u_{1}(\theta), u_{3}(\theta), \ldots,\right. \\
\left.u_{21-1}(\theta)\right) d \theta+e^{2 \pi t} \int_{0}^{2 \pi} e^{-a \theta} a_{1} u^{2 k+1}(\theta) d \theta, \\
1 \leq 1 \leq k
\end{gathered}
$$


Moreover by continuous dependence arguments there extst $c^{\infty}$ functions $8_{1}$ such that the solutions of $(4.5)$ can be written as

$$
\begin{aligned}
u_{21+1}(\theta)= & g_{1}\left(\theta, a_{0}, a_{1}, \ldots, a_{1}(2 \pi), u_{2}(2 \pi), \ldots, u_{21+1}(2 \pi)\right), \\
& 1 \leq 1 \leq k
\end{aligned}
$$

and

$$
u_{1}(\theta)=e^{a} \theta
$$

Define the functions $h_{1}$ as

$$
\begin{aligned}
& h_{1}\left(\theta, a_{0}, a_{1}, \ldots, a_{1-1}, u_{1}(2 \pi), u_{3}(2 \pi), \ldots, u_{21+1}(2 \pi)\right) \\
& =\left(f _ { 1 } \left(a_{1}, a_{2}, \ldots, a_{1-1}, e^{a_{0} \theta}, B_{1}\left(\theta, a_{0}, a_{1}, u_{1}(2 \pi), u_{3}(2 \pi)\right),\right.\right. \\
& \ldots, g_{1-1}\left(\theta, a_{0}, \ldots, a_{1-1}, u_{1}(2 \pi), u_{3}(2 \pi), \ldots, u_{21-1}(2 \pi)\right) \\
& 1 \leq 1 \leq k .
\end{aligned}
$$

Substituting the above $\ln (4.6)$ we have

$$
\begin{aligned}
& u_{1}(2 \pi)=e^{2 \pi a} \\
& u_{21+1}(2 \pi)=e^{2 \pi a_{0}} \int_{0}^{2 \pi} e^{-a_{0} \theta} h_{1}\left(\theta, a_{0}, a_{1}, \ldots, a_{1}-1, u_{1}(2 \pi),\right. \\
& \left.u_{3}(2 \pi), \ldots, u_{21-1}(2 \pi)\right) d \theta+e^{2 \pi a_{0}} \int_{0}^{2 \pi} a_{1} e^{21 a_{0} \theta} d \theta \\
& 1 \leq 1 \leq k .
\end{aligned}
$$


Now (4.7) gives an implicit relationship between the set $\left(u_{1}(2 \pi), u_{3}(2 \pi), \ldots, u_{2 k+1}(2 \pi)\right)$ and the set $\left(a_{0}, a_{1}, \ldots, a_{k}\right)$. Thus In order to show $\left(a_{0}, a_{1}, \ldots, a_{k}\right)$ is a continuous function of $\left(u_{1}(2 \pi), u_{3}(2 \pi), \ldots, u_{2 k+1}(2 \pi)\right)$ in netghborhood of $(1,0,0, \ldots, 2 \pi a)$ we apply the inverwe mappling theorem to $(4.7)$. Denoting the right side of (4.7) by $F_{21+1}$, we must prove that the determinant of the Jacoblan

$$
\left(\frac{\partial F_{21+1}}{\partial a_{j}}\right) \text { evaluated at }\left\{\begin{array}{c}
a_{1}=0,0 \leq 1 \leq k-1, a_{k}=a \\
u_{1}(2 \pi)=1, u_{21+1}(2 \pi)=0,1 \leq 1 \leq k-1, \\
u_{2 k+1}(2 \pi)=a
\end{array}\right.
$$

1s not zero. Since the matrix in (4.8) is lower triangular (from (4.7)) we easily have that 1 ts determinant evaluated at the point given in (4.8) 1s equal to $(2 \pi)^{k+1}$. In viaw of the smoothness of the functions $\mathrm{F}_{21+1}$ the proof of Proposition 4.1 is complete.

Proposition 4.2. Let $1, k$ be integers with $0 \leq j \leq k$. Assume $\bar{a}>0$ and $\lambda \neq 0$ and constder the function $f:[0, \overline{0}] \times[0, \bar{\theta}]+R$ defined as

$$
f(\mu, x)=(x-\mu)(x-2 \mu) \ldots(x-j \mu)(x+\mu)^{k-j}+\phi(\mu, x)
$$

where $\left.\phi(*, \cdot) \in c^{k+1}[10, \bar{\theta}] \times[0, \bar{a}], R\right]$. Suppose that for each $\mu \in[0, \bar{a}], \phi(\mu, \cdot)=O\left(x^{k}\right)$ and that the coeffictent of the $x^{k+1}$ term of the Maclaurin expansion of $\phi(0, \cdot)$ is not zero. Then there exists 
$x_{1}>0, \mu_{1}>0$ such that for $0<\mu<\mu_{1}, f(\mu, \cdot)$ has exactly $f$ zeros on the interval $\left(0, x_{1}\right)$ and they are all simple. Moreover these zeros lie on the interval $[0,(f+1) \mu]$.

Phoof: Without 108 of generallty we may asume that the coefficlent of the $x^{k+1}$ term of the Maclaurin expanaton of $\phi(0, *)$ is positive, for otherwise we may replace $f(\mu, x)$ with $-f(\mu, x)$. There exista $\mu_{2}>0, x_{2}>0$ such that $\phi(\mu, \cdot)$ is strictiy increasing on $\left[0, x_{2}\right]$ for all $\mu \in\left(0, \mu_{2}\right]$. Set

$$
\bar{f}(\mu, x)=\frac{f(\mu, x)}{(x+\mu)^{k-j}} \text { and } \bar{\phi}(\mu, x)-\frac{\phi(\mu, x)}{(x+\mu)^{k-1}} \text {. }
$$

In order to prove our result it suffices to find an $x_{1}>0$ and $\mu_{1}>0$ such that for $\mu \in\left(0, \mu_{1}\right), \bar{f}\left(\mu_{1} \cdot\right)$ has exactly $j$ zeros on $\left(0, x_{1}\right]$ which are all simple. We then show that these zeros lif on the Interval $\left(0,(j+1)_{\mu}\right]$

Write

$$
\bar{f}(\mu, x)=\psi(\mu, x)+\bar{\phi}(\mu, x)
$$

where

$$
\psi(\mu, x)=\lambda(x-\mu)(x-2 \mu) \ldots(x-j \mu)
$$

It follows from the hypotheses on $\phi(\mu, x)$ and $(4.9)$ that $\frac{\partial^{1} \Phi(0,0)}{\partial x^{1}}=0$ 
for $0 \leq 1 \leq f$ and that $\bar{\phi} \in \mathrm{C}^{j}[[0, \bar{B}] \times[0, \bar{\theta}], R]$; thus $\overline{\mathrm{f}} \in \mathrm{C}^{\mathfrak{f}}[[0, \bar{\theta}]$ $\times[0, \bar{a}], R]$. Since $\frac{\partial^{f} \psi(0,0)}{\partial x^{j}}+0$ then $\frac{\partial^{J} f(0,0)}{\partial x^{j}}+0$; hence there exists $\mu_{3}>0, x_{3}>0$ such that $\frac{\partial^{f} f\left(\mu_{1} x\right)}{\partial x^{j}} \neq 0$ for $\mu \in\left[0, \mu_{3}\right]$ and $x \in\left[0, x_{3}\right]$. By Rolle's Theorem we Immediately conclude that for $\mu \in\left[0, \mu_{3}\right], \bar{E}(\mu, *)$ has no more than $f$ zeros (counting multip1lc1ty) on the Interval $\left[0, x_{3}\right]$.

It thus sufflces to show that there exist $\mu_{1} \leq \mu_{3}$ and $x_{1} \leq x_{3}$ auch that for $\mu \in\left[0, \mu_{1}\right], \bar{E}(\mu, *)$ has at least $f$ distinct zeros on $\left(0, x_{1}\right)$ in order to conclude that $\bar{f}(\mu, \cdot)$ for $\mu \in\left[0, \mu_{1}\right]$ has exactly 1 rootg on $\left(0, x_{1}\right)$, which are all simple. From the conditions on $\phi(\mu, x)$ there exists an $x_{4}>0$ and $\mu_{4}>0$ with $x_{4} \leq x_{3}$ and $\mu_{4} \leq \mu_{3}$ such that $\bar{\phi}(\mu, \cdot)$ is strictly increasing on $\left[0, x_{4}\right]$ for each $\mu \in\left(0, \mu_{4}\right]$. Since $\psi(\mu, x)$ is a homogeneous polynomial in $\mu, x$ it follows that

$$
\psi(\mu, x)=\lambda\left(x^{\jmath}+c_{1} \mu x^{j-1}+c_{2} \mu^{2} x^{j-2}+\ldots c_{j} \mu^{j}\right)
$$

for approprlate constants $c_{1}, 1 \leq 1 \leq j$. We now obtain estimates on the ordinates of the $f$ relative extremum of $\psi(\mu, \cdot)$. Consider the roots of

$$
\frac{\partial \psi(\mu, x)}{\partial x}=\lambda\left(j x^{j-1}+c_{1}(j-1) \mu x^{j-2}+\ldots c_{k-1} \mu^{k-1}\right)=0 .
$$

For $u=1$ let $b_{s} 1 \leq s \leq j-1$ be the real solutiona of $(4,10)$ efnce $\psi(\mu, \cdot)$ has $f-1$ distinct relative extremum. Thus $b_{8}$ satisfy 


$$
f b_{a}^{j-1}+c(j-1) b_{a}^{j-2}+\ldots c_{j-1}=0
$$

and $b_{g}$ satiafies $(4.10) ;$ Indead

$$
\begin{aligned}
\frac{\partial \psi}{\partial x}\left(\mu, b_{a} \mu\right) & =\lambda\left(\mu^{j-1} j b_{a}^{j-1}+\mu^{j-1} c(j-1) b_{a}^{j-2}+\ldots \mu^{j-1} c_{j-1}\right) \\
& =\lambda \mu^{j-1}\left(\lambda b_{a}^{j-1}+c(j-1) b_{a}^{j-2}+\ldots c_{j-1}\right)=0,
\end{aligned}
$$

in view of (4.11). Thus for each $\mu$ the relative extremum of $\psi(\mu, \cdot)$ occur at $x=b_{g} \mu$ for $1 \leq a \leq 1$ and

$$
\psi\left(\mu, b_{s} \mu\right)=\lambda \mu^{j}\left(b_{s}^{j}+c_{1} b_{s}^{j-1}+\ldots c_{j}\right)
$$

From the properties of $\Phi(\mu, x)$ we observe that $\Phi(\mu$, m $)=0\left(\mu^{j}\right)$ for any number $m$. Let us now plck a number $\mu_{1}>0$ satisfylng several conditions. First of all select $\mu_{1}>0$ such that $\mu_{1}<\mu_{4}$ and $(j+1) \mu_{1} \leq x_{4}$. In addition let $\mu_{1}$ have the property that alI $\mu \in\left[0, \mu_{1}\right]$

$$
\begin{aligned}
& \text { (1) }\left|\psi\left(\mu_{s}, j\right)\right|>|\bar{\phi}(\mu, j \mu)| \quad s=1,2, \ldots, j \\
& \text { (11) } \mid \psi\left(\mu_{s}(j+1) \mu|>| \bar{\phi}(\mu,(j+1) \mu) \mid .\right.
\end{aligned}
$$

Such a $\mu_{1}$ exlote in view of $(4,12)$ and the fact that $\Phi(\mu, m)=0\left(\mu^{j}\right)$. For $\mu \in\left(0, \mu_{1}\right], \bar{\phi}\left(\mu_{*}\right)$ is increasting for $x \in\left[0,(j+1) \mu_{1}\right]$ (atnce $\left.(j+1) \mu_{1} \leq x_{4}\right)$ and thus from $(4.13)$ (1) $\left|\psi\left(\mu, b_{a} \mu\right)\right|>\left|\Phi\left(\mu, b_{s} \mu\right)\right|$. 
It thus follow for $0<u \leq \mu_{1}$ that on any Interval $(\ell \mu,(\ell+1) \mu)$, a pastlue integer $<j$, In which $\psi(\mu, \cdot)$ is negative then $\bar{f}(\mu, *)=\psi(\mu, *)+\bar{\phi}(\mu, *)$ has at least two zeros. Thus one of three possiblities can occur:

(a) $\bar{E}(\mu, *)$ has at least $j-2$ zeros on $(\mu, j \mu)$ and $\psi(\mu, *)$ is negative on $[0, \mu)$ and on $(j \mu,(f+1) \mu]$. But then $\bar{F}(\mu, \cdot)$ has at least one zero on $(0, \mu)$ alnce $\bar{\phi}(\mu, 0)=0$ and $\bar{\phi}\left(\mu_{*} \cdot\right)$ is postulve on $(0, \mu)$. Moreover, in vlew of $(4.13)(11), \bar{f}\left(\mu_{*} *\right)$ has at least one zero on $(J \mu,(1+1) \mu)$.

(b) $\vec{f}(\mu, \cdot)$ has at least $(j-1)$ zeros on $(\mu, j \mu)$ and etther $\psi(\mu, *)$ negative on $[0, \mu)$ or on $(f \mu,(f+1) \mu]$. Reagonirg as In (a) $\bar{f}(\mu, \cdot)$ had at least one zero on elther $(0, \mu)$ or on $(J \mu,(1+1) \mu)$.

(c) $\bar{f}(\mu, *)$ has at leas $j$ zeros on $(\mu, j \mu)$. In a11 three cages $\bar{f}(\mu, \cdot)$ has at least $f$ distinct zeros on $\left(0,(j+1) \mu_{1}\right)$ for each $\mu \in\left(0, \mu_{l}\right]$ and in fact these zerog $11 \mathrm{e}$ on $(0,(f+1) \mu)$. If let $x_{1}=(y+1) \mu_{1}$ we obtain the conclusions of Proposition 4.2 .

We now provide information concerning the zeros of $\tilde{V}(f, c)$ in a neighborhood of $\left(f_{0}, 0\right)$.

Phoposition 4.3. Assume the solution $x=y=0$ of the system (2.1) 1 elther h-asymptotically stable of h-completely unatable. Then there exists neighborhood $N$ af $E_{0}$ and a number $r_{3}, 0<r_{3}<r_{0}$ guch that for $f \in N, V(f, *)$ has at most $k$ zeros on $\left[0, x_{3}\right]$ where $k=\frac{k-1}{2}$ 
Phoof: As indicated before we may assume gystems (2.1) and (2.2) have the noma1 form $(4.3)$ and $(4.1)$ reapectively, For $c \neq 0, \ddot{V}(f, c)$ is given by

$\tilde{v}(f, c)=\left(u_{1}(E, 2 \pi)-1\right)+u_{3}(f, 2 \pi) c^{2}+\ldots y_{2 k+1}(f, 2 \pi) c^{2 k}+\tilde{\rho}(f, c)$

where $\tilde{\rho}(f, c)=\frac{\rho(\tilde{E}, c)}{c}$ and $\tilde{p}(f,+)$ had degree $>2 k$. By gubatfuting $z=c^{2}$ it then suffices to prove that $\tilde{V}(f, \sqrt{z})=0$ has at most $k$ roots. Now

$$
\tilde{V}\left(f_{0}, \sqrt{z}\right)=2 \pi a z^{k}+\tilde{\rho}\left(f_{0}, \sqrt{z}\right)
$$

where $a 0$ and $\tilde{\rho}\left(f_{0}, \sqrt{z}\right)=0\left(z^{k}\right)$. By cont1nuous dependence argument $\frac{\partial^{1} \tilde{v}}{\partial k}(f, \sqrt{z})$ is continuous for all $f$ in a nelghborhood of $f_{0}$, for al1 2 in a right nelghborhood of 0 , and for all integers 1 . since $\frac{\partial^{k} \tilde{v}}{\partial{ }_{2}}\left(f_{0}, 0\right)=2 \pi a \neq 0$ then by continuty there extst a neighborhood $N$ of $f_{0}$ and number $r_{3}$ auch that $\frac{\partial^{k} v}{\partial z^{k}}(f, \sqrt{z}) \neq 0$ for each $t \in N$ and each $z \in\left[0, x_{3}^{2}\right]$. An application of Rolle'g Theorem tello us that for each $E \in N$ there are at most $k$ roots of the equation $\tilde{V}(f, \sqrt{z})=0$ fot $z \in\left[0, r_{z}^{2}\right]$; that 1 for each $f \in N$ there are at most $k$ roots of the equation $\tilde{V}(f, c)=0$ for $c \in\left[0, x_{3}^{2}\right]$. This concludes the proof of the proposition.

Proposition 4.4. Assume the solution $x \equiv y \equiv 0$ of (2.1) 1s elther h-asymptoticaliy atable or h-completely unatable. There exists 
number $r_{4}, 0<r_{4}<r_{0}$ auch that for any $r E\left(0, r_{4}\right)$ there exista a neighborhood $N_{r} \subseteq N$ so that for any $f \in N_{r}, \tilde{V}(f, \cdot)$ has no zeros for $c \in\left[r, r_{4}\right]$.

Proof: Consider

$$
\tilde{v}\left(f_{0}, c\right)=2 \pi a c^{2 k}+\tilde{\rho}\left(E_{0}, c\right)
$$

where $\tilde{\rho}\left(f_{0}, c\right)=0\left(c^{2 k}\right)$. It follows immedlately that there extats a number $\mathbf{r}_{4}, 0<\mathbf{r}_{4}<\mathbf{r}_{0}$ such that

$$
\left|\tilde{v}\left(E_{0}, c\right)\right|>\pi|a| e^{2 k} \text { for } c \in\left[0, x_{4}\right] \text {. }
$$

Now given any $x \in\left(0, r_{4}\right]$

$$
\left|\tilde{V}\left(f_{0}, c\right)\right|>\pi|a| r^{2 k} \text { for } c \in\left[r, x_{4}\right]
$$

Because of the continutty of $\tilde{v}(\cdot, *)$ and the compactness of $\left[r, r_{4}\right]$ it follows that there extsts a neighborhood $N_{r}$ of $f_{0}$ with $N_{r} \subseteq N$ such that for $f \in N_{r}$ and for ald $c \in\left[x, r_{4}\right]$

$$
|\tilde{V}(f, c)-\tilde{V}(f, c)|<\frac{1}{2} \pi|a| r^{2 k}
$$

Thus from $(4.14)$ and $(4.15)$ for $f \in N_{x}$ and $c \in\left[r, r_{4}\right]$, $v(f, c)>\frac{1}{2} \pi|a| r^{2 k}$, completing the proof of the propostition. We now prove the necesalty part of theoren 3.1 , that 1s we prove 
that the h-asymptotic stablifty or h-complete instablifty of the Bolution $x \equiv y \equiv 0$ of (2.1) with $h=2 k+1$ 1mplles (1)-(1v) hold. We then prove Theorem 3.2, the guffictency part of the Theorem 3.1 , and Theorem 3.3 .

\section{Phoof:}

(1) Choose $r_{1}=\min \left\{r_{3}, r_{4}\right\}$. Then the result follows inmediately Erom Proposition 4.3 since the nonzero roots of $\tilde{v}(f, 0)=0$ correspond to the nontrivial perlodic solutions of $(4.1)$,

(11) In oraer to prove the result we clafin it 18 permisubible to once again assume that $(2.1)$ and $(2.2)$ are In normal form. Indeed, we prove that for any integer $j, 0 \leq j \leq k$ and for any neighborhood $B^{2}\left(r_{2}\right)$ or the origln, $r_{2} \in\left(0, x_{1}\right)$, there extats a two parameter fandly of functions $f_{\mu, t}^{N}\left(x_{1}, y_{1}\right)$ (racal1 $f^{N}\left(x_{1}, y_{1}\right)=(T * f)\left(x_{1}, y_{1}\right)$ and we replace $\left(x_{1}, y_{1}\right)$ with $\left.(x, y)\right)$ such that for l,t sufficlently gma11, sygtem (4.2) has exactly $f$ nontrivial perlodic solution in $B^{2}\left(r_{2}\right)$ and no pertodtc solutlons $\mathrm{tn} \mathrm{B}^{2}\left(\mathrm{x}_{1}\right) / \mathrm{B}^{2}\left(\mathrm{r}_{2}\right)$. Moreover $f_{\mu, t}^{N}(*) \rightarrow f^{N}()$ as $(\mu, t)+(0,0)$. Since $f_{\mu, t}^{N}$ is a two parameter family than the corresponding Takeng" transtormation of this family can be consldered as a $C^{+0}$ diffeomorplutan $T: R^{2} \times R^{2}+R^{2} \times R^{2}$; that is $d=2$. More percisely, $\left(\begin{array}{c}\mu \\ \tau \\ x \\ y\end{array}\right)=\tau^{-1}\left(\mu, \tau, x_{1}, y_{1}\right)$ and $f_{\mu, \tau}(x, y)=$ $\left(T^{*}\left(\mu, t, x_{1}, y_{1}\right)^{-1} f_{\mu, \tau}^{N}\right)\left(x_{1}, y_{1}\right)$, and thus $f_{\mu, t} \rightarrow f$ in the topology deacribed in the beglnning of Section 2 . Moreover for each $(\mu, \tau)$, 
$\left(T^{-1}(\mu, t, \cdot, \cdot)\right.$ Is a homeomorphism of a neighborhood of the origin in $\left(x_{2}, y_{1}\right)$ apace onto nelghborhood of the ox1gin in the $(x, y)$ space. Fron these considerations it follows that for each integer $f$, $0 \leq 1 \leq k$, for each nelghborhood $N^{*}$ of $f_{0}$, and for each $t \in\left(0, r_{1} 1\right.$ there exists $(\mu . \tau)$ sufficlently amall such that $f_{\mu, \tau} \in N^{*}$ and aysten $(2.1)$ has $f$ perfodic orbits in $B^{2}(r)$.

It thus suffices to prove our assertions given in the beginning of the proof and we may assume that (2.1) and (2.2) are in normal form. Proceeding with the proof, pick any integer $j, 0 \leq j \leq k$, any neighborhood $N^{*}$ of $E_{0}, N^{*} \subseteq N$ and any number $x_{2} \in\left(0, r_{1}\right]$. Now let $\mu$ be a postive constant and we claim we can select a one paraweter fandly of functions $f_{\mu}$ auch that $E_{\mu}+f_{0}$ as $\mu+0$ and $\tilde{v}\left(f_{\mu}, c\right)$ can be written as

$$
v\left(f_{\mu}, c\right)=2 \pi a\left(c^{2}-\mu\right)\left(c^{2}-2 \mu\right), \ldots,\left(c^{2}-j \mu\right)\left(c^{2}+\mu\right)^{k-j}+\tilde{\rho}(\mu, c),
$$

where $\tilde{p}(\cdot, \cdot) \in c^{\infty}$ and $\tilde{p}(\mu, c)=O\left(c^{2 k}\right)$. Indeed from Propostition 4.1 there exists $b_{1}>0$ such that for $\mu \in\left[0, b_{1}\right]$ we can select $a_{1}(\mu)$, $1 \leq 1 \leq k, \ln (4,1)$ so that the $2 k^{\text {th }}$ order Taylor expanstion of $\tilde{\mathrm{v}}\left(f_{\mu}, \cdot\right)$ has exactly the form

$$
2 \pi a\left(c^{2}-\mu\right)\left(c^{2}-2 \mu\right), \ldots,\left(c^{2}-j \mu\right)\left(c^{2}+\mu\right)^{k-j} .
$$

Moreover we may gelect $f_{\mu}$ so that $\ln (4.1)$ 


$$
\begin{aligned}
& \Phi\left(f_{\mu}, x, y\right) \equiv \phi\left(f_{0}, x, y\right) \\
& \Psi\left(f_{\mu}, x, y\right) \equiv \Psi\left(f_{0}, x, y\right) .
\end{aligned}
$$

In view of the Takens normal form given in (**) we may assume that

$$
\begin{aligned}
& \Phi\left(f_{\mu}, x, y\right) \equiv \Phi\left(f_{0}, x, y\right)-a_{k+1} x\left(x^{2}+y^{2}\right)^{k+1}+\hat{\Phi}\left(f_{0}, x, y\right) \\
& \Psi\left(f_{\mu}, x, y\right) \equiv \Psi\left(f_{0}, x, y\right)=a_{k+1} y\left(x^{2}+y^{2}\right)^{k+1}+\hat{\Psi}\left(f_{0}, x, y\right),
\end{aligned}
$$

where $\hat{\Phi}\left(f_{0}, x, y\right), \hat{\varphi}\left(f_{0}, x, y\right)=0\left(\left(x^{2}+y^{2}\right)^{k+2}\right)$. Then (4.16) takes the form

$$
\begin{gathered}
\tilde{V}\left(f_{\mu}, c\right)=2 \pi a\left(c^{2}-\mu\right)\left(c^{2}-2 \mu\right), \ldots,\left(c^{2}-j \mu\right)\left(c^{2}+\mu\right)^{k-j}+ \\
u_{2 k+3}\left(f_{\mu}, 2 \pi\right) c^{2 k+3}+\rho^{*}(\mu, c),
\end{gathered}
$$

where $\rho^{*}(\mu, c)=0\left(c^{2 k+2}\right)$ and $u_{2 k+3}\left(f_{\mu}, 0\right)$ corresponds to the coefficlent of $c^{2 k+3}$ in the expansion of $r$ (see (2.4)) for the system given by $E_{\mu}$. Uatigg $(4.3)$ and $(4.18)$ one find that $u_{2 k+3}\left(f_{0}, 0\right)$ $=a+1^{\theta}$.
We whin to apply Propostition 4.2 to the function $\frac{\tilde{V}\left(f_{\mu}, c\right)}{\left(c^{2}+\mu\right)^{k-j}}$ with $x=c^{2}$. This requites $0 \neq u_{2 k+3}\left(f_{0}, 2 \pi\right)=2 \pi a_{k+1}$. Hence if $a_{k+1} \neq 0$ we are in position to apply Proposition 4.2 . If $a_{k+1}=0$ then define for $\tau \in(0,1]$ 


$$
E_{\mu, \tau}(x, y)=f_{\mu}(x, y)+\tau x\left(x^{2}+y^{2}\right)^{k+1}
$$

So as not to have to d1scuss the two casea $a_{k+1}=0$ and $a_{k+1} \neq 0$ separately let us define for all $t \in(0,11$

$$
\tilde{E}_{\mu, \tau}(x, y) \equiv f(x, y)
$$

In case $a_{k+1} \neq 0$. Now write the function $\tilde{V}\left(f_{\mu, \tau}, c\right)$ as

$$
\begin{gathered}
\left.\tilde{v}\left(f_{\mu, \tau}, c\right)=2 \pi a\left(c^{2}-\mu\right)\left(c^{2}-2 \mu\right), \ldots,\left(c^{2}-j \mu\right)\left(c^{2}+\mu\right)\right)^{k-1}+ \\
u_{2 k+3}\left(f_{\mu, \tau}, 2 \pi\right) c^{2 k+2}+\hat{p}(\tau, \mu, c)
\end{gathered}
$$

where

$$
u_{2 k+3}\left(E_{0, \tau}, 2 \pi\right)=\left\{\begin{array}{lll}
2 \pi t & \text { if } & a_{k+1}=0 \\
2 \pi a_{k+1} & \text { if } & a_{k+1} \neq 0
\end{array},\right.
$$

and $\hat{p}(\tau, \mu, c)=O\left(c^{2 k+2}\right)$. Deflning (as in the proof of Proposttion 4.2)

$$
\hat{V}(\mu, \tau, x)=\frac{\tilde{V}\left(f_{\left.\mu, \tau^{, \sqrt{x}}\right)}\right.}{(x+\mu)^{k-j}}
$$

we need to show that there exists $(\mu, \tau)$ sufftciently sma11 so that $f_{\mu, \tau} \in N^{*}$ and $\hat{V}(\mu, \tau, *)$ has exactly $f$ zeros on the interval $\left(0, r_{2}^{2}\right]$ $\left(\operatorname{recal1} x=c^{2}\right)$. Now $\frac{\partial^{j} V(0,0,0)}{\partial x^{3}} \neq 0$ and by the continutity of 
$\frac{\partial^{\dagger} V}{\partial x^{j}}(*, *)$ there exlats $\bar{\mu}, \bar{T}, x$ with $x_{1} \leq \tau_{2}^{2}, \mu \leq b$ such that $\frac{\partial^{J} \hat{V}(\mu, \tau, x)}{\partial x^{j}} \neq 0$ for $x \in\left(0, x_{1}\right], \tau \in[0, \tau]$, and $\mu \in[0, \bar{\mu}]$. As a consequence of Rolle's Theorem, for $\tau \in[0, \bar{T}], \mu \in[0, \bar{\mu}]$ and $x \in\left[0, x_{1}\right], \hat{v}(\mu, t, \cdot)$ has at most $f$ zeros on the Interval $\left[0, x_{1}\right]$. From the continusty of $u_{2 k+3}\left(\varepsilon_{\mu, \tau}, 2 \pi\right)$ in $\mu$ and $\tau$ and the fact that ${ }_{2 k+3}\left(F_{0, \tau}, 2 \pi\right) \neq 0$ (see $\left.(4.21)\right)$ for $t \in(0, T]$, then for each $T \in(0, \bar{T}]$ there extsts a number $\mu_{1}(\tau)$ such that for $\mu \in\left[0, \mu_{1}(\tau)\right]$ $u_{2 k+3}\left(E_{\mu, t}, 2 \pi\right) \neq 0$.

since $E_{\mu, \tau} \rightarrow F_{0}$ as $\mu, \tau \rightarrow(0,0)$ we may plck $\tau_{1} \leq \tau$ and $\mu^{*} \leq \operatorname{mnn}\left(\mu_{1}\left(\tau_{1}\right), \mu\right)$ so that for $\mu \in\left[0, \mu^{*}\right]$

$$
\Sigma_{\mu_{1} \tau_{1}} E N_{x_{1}} \cap N^{*}
$$

where $N_{x_{1}}$ is given in proposition 4.4 . Thus $\hat{v}\left(\mu, \tau_{1}, \cdot\right)$ has no zeros on $\left[x_{1}, x_{1}^{2}\right]$ for a.1 $\mu \in\left[0, \mu^{*}\right]$.

Applying Proposition 4.2 to the function $\hat{v}(\mu, \tau, x)$, there existo $\mu_{2}<\mu^{*}$ with $(J+1) \mu_{2} \leq x_{1}$ such that for $\mu \in\left[0, \mu_{2}\right], \hat{V}\left(\mu, T_{1}, \cdot\right)$ has $f$ distinct zeros on the interval $(0,(j+1) \mu)$. Since for $\mu \in\left[0, \mu_{2}\right], V\left(\mu, \tau_{1},\right)$ has at moat $j$ zeros on $\left(0, x_{1}\right]$ we conclude $\hat{v}\left(\mu, \tau_{1}, \cdot\right)$ has exactly $f$ positive zeros on $\left(0, x_{1}\right]$ and no zeros on $\left[x_{1}, r_{2}^{2}\right]$. Since $x_{1} \leq t_{2}^{2}, E_{\mu, \tau_{1}} \in N^{k}$, and $V\left(f_{\mu, \tau_{1}}, \cdot\right)$ has exactly $j$ distinct zeros on $\left(0, \mathrm{r}_{2}\right)$, the proof of (11) is complete. (1i1) From (1) and (11) it follows that $N=\bigcup_{j=0} s_{j}$ is nonempty for each $j, 0 \leq j \leq k$. Now choose any $f$ with $0 \leq j \leq k$ and we sha11 
show that the thterior of $\mathrm{s}_{\mathrm{g}}$ is not empty. Consider the functions $F_{\mu, \tau} \in S_{y}$ conatructed $\ln (11)$ and we show for $\mu$ and $t$ sufficlently smal1 $E_{\mu, t} 19$ in the interior of $s_{j}$. As in the proof of $(11)$, an application of Rolle' Theoren Implles that there extst $b_{1} \leq r_{1}$ and a nelghborhood $N_{1}$ of $f_{\mu, t}$ Eot all $\mu, t$ suffictently sma11, $N_{2} \subset N$, such that for $f \in N_{1}, \ddot{v}\left(f,{ }^{\circ}\right)$ has at most $I$ zeros on $\left[0, b_{1}\right]$. From proposition 4.4 there exista a nelghborhood $N_{b_{1}}$ of $E_{0}$, $N_{b_{1}} \subset N$ ao that for $f \in N_{b_{1}}, \tilde{V}\left(f_{*},\right)$ has no zeros on $\left[b_{1}, r_{1}\right]$. Plok $\mu_{s} t$ so small thet $f_{\mu, \tau} \in N_{b_{l}} \cap \mathbb{N}$ and the zeroe of $V\left(f_{\mu, T^{*}}\right)$ lie in $\left[0, \frac{b_{1}}{2}\right]$. Since $V\left(F_{\mu, r^{*}}\right)$ has $J$ almple zeros on $\left[0, \frac{1}{2}\right]$ there exlats neighborhood $N_{2}$ of $f_{\mu_{1}}, N_{2} \subset N$ wo that for $f \in N_{2}, \tilde{V}(E$,$) has at least \bar{j}$ zeros on $\left[0, b_{1}\right]$. Defining $N_{3}=N_{1} \cap N_{b} \cap N_{2}$, we see that $N_{3}$ la nonempty nelghborhood of $H_{1} T$ and for $f \in N_{3}, \tilde{V}(E, \cdot)$ has exactly $\rfloor$ zeros on $\left[0, b_{1}\right]$ and no zeros on $\left[b_{1}, x_{1}\right]$; that is $f \in S_{f}$. Thus $f_{\mu, t}$ is in the intertor of $S_{f}$ for all $\mu, t$ sufficientiy anal1. Since $f_{\mu, t} \rightarrow f_{0}$ as $(\mu, t) \rightarrow(0,0)$ then $f_{0}$ La in the clogure of the Intertor of $s_{j}$; that is $f_{0}$ is or the bouncary of $\mathfrak{S}_{j}$ for each $\bar{j}, 0 \leq \bar{J} \leq k$. This concluces the roor.

(iv) Thls follows frmediately from Proposteton 4.4

Remarks. We observe thet in order to construet if lylng in the intertor of 5 , we con replace the polynomial part of order $2 \mathrm{k}$ in $(4.16)$ with the more gereral expresston

$$
2 n a\left(c^{2}-\mu_{1}\right)\left(c^{2}-\mu_{2}\right), \ldots,\left(c^{2}-\mu_{1}\right)\left(c^{2}+\alpha\right)^{k-1}
$$


where $\mu_{1}, 1 \leq i \leq f$, and $\alpha$ are oufficlently stal1 posttive numbers and the $\mu_{f}^{\prime} 8$ are distinct. From Propogition 4.1 we have a $1-1$ correspondence between the coefficlents of the polynomial part of order $k$ of $\tilde{V}(f, \sqrt{c})$ and the coefficlents of the right-hand stde of $(4.1)$ (up to order $2 k+1$ ). Moreover small perturbations (of order $k+1$ ) of the polynomials of order $k$ having $f$ almple zeros will not caube any change in the number of zeros of $\widetilde{v}(\tilde{f}, \sqrt{c})$. Thus functions $f$ can easily be constructed that preserve the number of periodic orbits under perturbation.

We also see that if $f \in S_{k}$ then $\tilde{V}(E, \sqrt{c})$ has exactly $k$ simple roots in a sufficlently mall nelghborhood of the origin, It then follows that $f$ in the interior of $s_{k}$, that $18, s_{k}$ ls open.

Proof of Thearem 3.2 .

Necessity: Constder the following perturbation of (2.1)

$$
\begin{aligned}
& \dot{x}=-y+x_{0}(x, y)-b x\left(x^{2}+y^{2}\right)^{j} \\
& \dot{y}=x+Y_{0}(x, y)-b y\left(x^{2}+y^{2}\right)^{j},
\end{aligned}
$$

where $b$ is a suffelently small postelve constant so that the righthand afde of syatem (4.22) 1tes in $N$. Let us now apply the polncaré procedure to systems $(2.1)$ and $(4.22)$. Since the solution $x \equiv y \equiv 0$ of (2.1) 1s nelther h-asymptotically atable not h-completely unatable for any $h>0$ there exlots $F$ of the form (2.6) with $m=2 j+2$ auch

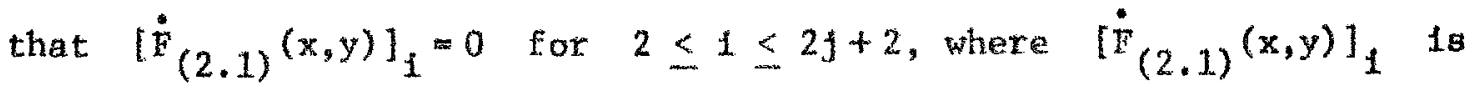
the homogeneous polynomial of degree 1 in the expanaton of $\dot{\mathrm{F}}_{(2,1)}$. Thus obtain 


$$
\dot{F}_{(4.22)}(x, y)=-b\left(x^{2}+y^{2}\right)^{j+1}+0\left(x^{2 j+2}\right)
$$

From proposition 3.1 we Imnediately conclude that the solution $x \equiv y \equiv 0$ of aystem $(4.22)$ 18 $2 j+1$-asymptotically gtable. From (11) of Theorem 3.1 applited to $(4.22)$ there exilatan $f \in N$ such that aystem (2.2) has $f$ perfodic orbits in $B^{2}(x)$. Thus we obtain the necessity part of Theorem 3.2 .

Subficiency: This follows immediately from the necessity part of Theorem 3.1 ( 1 ). Indeed if there axtats an integer $\bar{h}$ (which must be odd) for which the origin of $(2.1)$ is elther h-asymptotica11y stable or $\bar{h}$-completely ungtable then Theorem 3.1 (1) fmples that for all $f \in N$ there exist at nost $\frac{\bar{h}-1}{2}$ nontrivial periodic solutions of (2.2) lying in $B^{2}\left(x_{1}\right)$. Thts 19 contradiction and completes the proof of Theorem 3.2 .

Proof of the Sufficiency part of Theorem 3.1. We show that (1) and (11) Imply that the solution $x \equiv y \equiv 0$ of $(2.1)$ fa elther h-agymptotically stable on h-completely unstable where $h=21+1$. Applying the Poincare procedure to (2.1) we canclude that the solution $x \equiv y \equiv 0$ of (2.1) is either

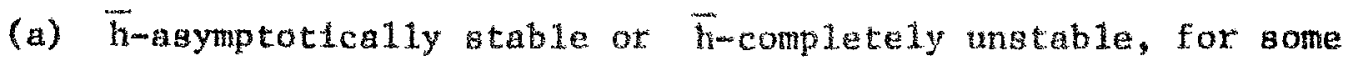
fintte $\overline{\mathrm{h}}$; or

(b) nether h-asymptotically stable nor hacomoletely unstable for any integer $\overline{\mathrm{h}}>0$.

In case (b), Theorem 3.2 Inplies that there exists an $f \in N$ such that equation $(2.2)$ has $k+1$ nontelvial pertodic solutions in $B^{2}(r)$. 
This contradicte (1). It thus remalns to prove $\bar{h}=\mathrm{h}$ in cage $(a)$. Let $\bar{n}=2 \pi+1$ and Elrst assume $\bar{k}>k$. By ualng the necessity part of Theorem 3.1 (11) (which we have already proved) there extats an $r \leq r_{1}$ and an $f \in N$ such that $(2.2)$ has $\bar{k}$ periodlc orbits in $\mathrm{B}^{2}(\mathrm{r})$. This contradicta (1). If $\overline{\mathrm{k}}>\mathrm{k}$ then again using the necessity part of Theoren 3.1 (1) there exista a nelghborhood $N$ af $f_{0}$ and a number $\bar{F}>0$ such that $f o x \in N$, system $(2.2)$ hat at most $\bar{k}$ nontrivial perlodic solutions in $p^{2}(r)$. Wut this contradicts Theorem 3.1 (11) aince there exist $F \in N$ and an $r<F$ for which system (2.2) has $k$ nontrivial perfodic solutlons $t$ th $B^{2}(r)$. Thls conpletes the proof.

Proof of Theorem 3.3. Choose $r_{3}$ and $N$ as theorem 3.1 (1) and whout loss of generality assume $G_{4}<0$.

We now Indicate a proot of the existence of a neighborhood $\mathrm{N}_{2}$ of $E_{0}, \quad N_{2} \subseteq N$, and a number $E_{2} \in\left(0, F_{1}\right)$ such that for each $E \in N_{2}$ whth $\alpha \leq 0$ there are no perlodlc orbtte of $(2.2)$ in $B^{2}\left(\varepsilon_{2}\right)$. Indeed It is sufficlent to prove that $\mathbb{N}_{2}$ and $E_{2}$ can be found so that $B^{2}\left(E_{2}\right) /(0,0)$ is a subset of the reglon of attraction of (2.2) for $f \in N_{2}$ and $\alpha \leq 0$. This easily follows by continulty arguments and an approprlate use of the polncare procedure in order to construct posttive deflnite functions (depending on f) which are negative definte in $B^{2}\left(e_{1}\right)$ along the aolutions of $(2.2)$ for $t \in N_{2}$. We leave the detalls to the reader.

Now choose $\bar{\varepsilon} \in\left(0, \varepsilon_{2}\right)$ and a netghorhood $\bar{N}$ of $\tilde{f}_{0}, \bar{N} \subset N$, such that the origin te the only critical point of $(2.2) \ln \mathrm{B}^{2}(\varepsilon)$ for each $f \in \bar{N}$. Stnce the or Igin of syotam (2.1) la abymptotically stable 
then the origin is totally stable. Now pick any $\varepsilon_{1} \in(0, \bar{E})$. Then the total stab111ty implies that thexe exist $\delta_{1}=\delta_{1}\left(\varepsilon_{1}\right)$ and a nefghborhood $N_{1} \equiv N_{1}\left(c_{1}\right)$ of $f_{0}, N_{1} \subset \bar{N}$, such that for every $\left(x_{0}, y_{0}\right) \in B^{2}\left(\delta_{1}\right)$ and $f \in N_{1}$ the positive orbit of $(2.2)$ passing through $\left(x_{0}, y_{0}\right)$ is contalned $\mathrm{kn} \mathrm{B}^{2}\left(\varepsilon_{0}\right)$. Let us assume $f \in \mathbb{N}_{1}$ and $\alpha>0$. Then if $\left(x_{0}, y_{0}\right) \in B^{2}\left(\delta_{1}\right)$ the postitve 1lmte set of the orbit pasaing through $\left(x_{0}, y_{0}\right)$ la nonempty, Ltes in $\overline{B^{2}\left(\varepsilon_{1}\right)}$, and contains no critlcal points (the ordgln is repelifing for (2.2) since $\alpha>0)$. By the well known Bendixaon Theoren [9] the positive 1fmlt set is a perlodic orbit. It 18 the only perlodic orbit contained in $\mathrm{B}^{2}\left(\mathrm{r}_{1}\right)$ in virtue of Theorem $3.1(1)$.

The proof of Theoten $3.31 \mathrm{~s}$ complete if we define $\mathrm{N}=\mathrm{N}_{1} \cap \mathrm{N}_{2}$ and choose any $\varepsilon \in\left(\varepsilon_{1}, \vec{\varepsilon}\right)$. 


\section{RETERENCES}

(1) Andronov, A. A., Leontovich, I. A., Cordon, I. I. and Mater, A. G., Theory of Bifurcation of Dynamic Syatems on a Plane, Halsted Press, New York (1973).

[2] Bernfeld, S. and Salvadord, L. Generalized Hopf Bifurcation, Proceedings of a Conference at Trleste, Italy (to appear).

[3] Chafee,N., Generalized Hopf H1furcation and Perturbation In a Ful1. Neighborhood of a Given Vector Fleld, Indlan Univ. Math. Journal 27 (1978), 173-194.

[4] Hale, J., Ordfnary Dlfferential Equations, Interscience, New York (1969).

[5] Hopf, E., Abzwelgung efner pextodischen lösung von einer stationären lösung eines differential systens, Ber Verh. Sach. Akad. Wias Leipsig Math. Nat., $94(1942), 3-22$.

[6] Marchett1, F., Negrln1, P., Salvador1, L., and Scalla M., LLapunov Direct Method in Approaching Blfurcation Problems, Ann. Mat. Pur. Appl., (1v) (cv111) (1976), 211-225.

[7] Marsden, I. E., and McCracken, M., The Mopf Bifurcation and 1 to App11catLong, Springer-Vexlag, New York, (1976).

[8] Negrin1, P., and Salvador1, L., Attractivity and Hopf bifurcatlon, Non1. Ana1., Theory, Math. and App1. 3(1979), 87-99.

19] Sansone, G. and Contl, R., Nonlineat Differential Equations; Macmillan, New York, 1964.

[10] Takens, F., Unfolding of Certaln Singulartites of Vector Fields: Generalized Hopf Bifurcations, J. Diff. Equas., 14(1973), 476-493. 